\begin{tabular}{|c|l|}
\hline Title & $\begin{array}{l}\text { Nitric Oxide Produced During Sublethal Ischemia Is Crucial for the Preconditioning-Induced Down-Regulation of } \\
\text { Glutamate Transporter GLT-1 in Neuron/A strocyte Co-Cultures }\end{array}$ \\
\hline Author(s) & Yamada, Takeshi; Kawahara, Koichi; Kosugi, Tatsuro; Tanaka, Motoki \\
\hline Citation & $\begin{array}{l}\text { Neurochemical Research, 31/1), 49-56 } \\
\text { https://doi.org/10.1007/311064-005-9077-4 }\end{array}$ \\
\hline Issue Date & 2006-01 \\
\hline Doc URL & http://hdl.handle.net/2115/5814 \\
\hline Rights & The original publication is avail lable at www.springerlink.com \\
\hline Type & article (author version) \\
\hline File Information & NR31-1.pdf \\
\hline
\end{tabular}

Instructions for use 


\title{
Nitric oxide produced during sublethal ischemia is crucial for the preconditioning-induced down-regulation of glutamate transporter GLT-1 in neuron/astrocyte co-cultures.
}

\author{
Takeshi Yamada, Koichi Kawahara, Tatsuro Kosugi, and Motoki Tanaka \\ Laboratory of Cellular Cybernetics, Graduate School of Information Science and \\ Technology, Hokkaido University, Sapporo 060-0814, Japan
}

Running head: nitric oxide and preconditioning-induced ischemic tolerance

Address correspondence to:

Koichi Kawahara, Ph D.

Professor of the Laboratory of Cellular Cybernetics,

Graduate School of Information Science and Technology,

Hokkaido University, Sapporo 060-0814

Japan.

E-mail: kawahara@cellc.ist.hokudai.ac.jp 


\begin{abstract}
In the brain, prior sublethal ischemia (preconditioning, PC) produces tolerance of neurons to subsequent lethal ischemia. This study aims at elucidating whether and how nitric oxide (NO) produced during PC is involved in the PC-induced ischemic tolerance of neurons in neuron/astrocyte co-cultures. The rise in the extracellular concentration of glutamate during ischemia caused by the reversed uptake of glutamate (Glu) by the astrocytic Glu transporter GLT-1 was markedly suppressed by the prior PC treatment, but the suppression was reversed by treatment with an inhibitor of nitric oxide synthase (NOS) during PC. Immunocytochemical and Western blot analyses demonstrated that the expression of GLT-1 was down-regulated after the PC insult, and this down-regulation was also antagonized by treatment with NOS inhibitors during PC. Here we show that nNOS-derived NO produced during PC was crucial for the down-regulation of astrocytic GLT-1, and this down-regulation coincided with an increased survival rate of neurons.
\end{abstract}

Key words nitric oxide, GLT-1, preconditioning, ischemic tolerance 


\section{Introduction}

Preconditioning (PC) to ischemic tolerance is a phenomenon in which a brief subtoxic insult induces robust protection against the deleterious effects of a subsequent, prolonged, lethal ischemia (1). In the brain, Kitagawa et al. (2) first reported that gerbils subjected to sublethal transient global ischemia exhibited reduced hippocampal neuronal death after a severe ischemic insult 24-28 h later. We have recently demonstrated in mixed neuron/astrocyte cultures that the ischemia-induced rise in the extracellular concentration of L-glutamate (Glu) is significantly suppressed by prior PC treatment, and this suppression during ischemia primarily contributes to the PC-induced ischemic tolerance of neurons (3).

Previous studies including by us have demonstrated that the astrocytic Glu transporter GLT-1 is primarily responsible for the clearance of extracellular Glu (4-7). These findings have led to the notion that a normal functioning astrocytic Glu transporter is crucial to the survival of neurons in the brain. Under conditions of brain ischemia, however, the role of astrocytic Glu transporters is reversed; that is, Glu is released from astrocytes to the extracellular space $(8,9)$, resulting in a massive excitotoxic death of neurons.

We have previously revealed that the production of nitric oxide (NO) is 
increased during PC, and this enhanced NO production is crucial to the development of PC-induced neuronal ischemic tolerance in cultured neurons (10). In addition, we have recently revealed that in neuron/astrocyte co-cultures the expression of the astrocytic glutamate transporter GLT-1 is transiently down-regulated after PC treatment, and the ischemia-induced rise in the extracellular concentration of glutamate via a reversed uptake of glutamate by GLT-1 is significantly suppressed (3). The question then arises as to whether NO produced during PC is involved in the PC-induced down-regulation of GLT-1, and in the resultant ischemic tolerance of neurons. In the present report, we provide experimental evidence that nNOS-derived NO produced during PC was crucial for the down-regulation of GLT-1, and for the increased neuronal resistance to subsequent severe ischemic insult. 


\section{Experimental Procedure}

The animal experiments conformed to the "Principles of laboratory animal care" (NIH publication No. 85-23, revised 1996), as well as the "guide for the care and use of laboratory animals”, Hokkaido University School of Medicine.

\section{Neuron/astrocyte co-culture}

Culture methods were described previously in detail $(3,4,10,11)$. In brief, both neurons and astrocytes were prepared from 17-day old embryonic rat cortices and grown in Dulbecco’s modified Eagle’s medium (DMEM, Gibco, Grand Island, NY) which was supplemented with 10\% heat-inactivated fetal bovine serum (FBS), 10\% Ham's F12, and 0.24\% penicillin/streptomycin (culture medium). Cells were plated at a uniform density of $3.0 \times 10^{5}$ cells/ $\mathrm{cm}^{2}$ onto poly-L-lysine $(100 \mu \mathrm{g} / \mathrm{ml})$-coated plastic dishes and maintained in a $5 \% \mathrm{CO}_{2}$ incubator at $37{ }^{\circ} \mathrm{C}$. The cultures were fed a filtered (0.22 $\mu \mathrm{m}$; Millipore, Bedford, TX) conditioned medium (CM) twice a week. To obtain the CM, cells from 16-18 day old embryonic rat cortices were plated onto poly-L-lysine-coated 6-well dishes and cultured for more than 2 weeks. The cultures were then fed a cooled culture medium and incubated for an additional day. The culture medium was filtered and used as CM. After 13-15 days, the neurons in these 
cultures sit on the top of a confluent monolayer of astrocytes. The experiments were performed using these cultures.

\section{Immunocytochemistry}

Astrocytes and neurons were identified by immunostaining with antibodies against glial fibrillary acidic protein (GFAP; Sigma, St Louis, MO) and microtubule-associated protein 2 (MAP-2; Sigma), respectively. The astrocytic glutamate transporter GLT-1 was detected by immunostaining with anti-GLT-1 polyclonal antibodies (Chemicon, Temecula, CA). The nitric oxide synthases (NOSs) in cultured cells were detected by immunostaining with anti-neuronal NOS (nNOS) (Euro-Diagnostica, Sweden), anti-endothelial NOS (eNOS) (Transduction Lab., Lexington KY), or anti-inducible NOS (iNOS) (Transduction Lab.). For the labeling of MAP-2, GFAP, GLT-1, nNOS, iNOS, and eNOS, the cortical cells were fixed with 4\% paraformaldehyde for 5 minutes at $4{ }^{\circ} \mathrm{C}$, followed by $95 \%$ methanol in PBS for 10 minutes at $-20{ }^{\circ} \mathrm{C}$. The cells were then incubated with a primary antibody over a $24 \mathrm{~h}$ period using a dilution of 1:1000 for MAP-2, 1:400 for GFAP, 1:2500 for GLT-1, 1:1,000 for nNOS, 1:1,000 for eNOS, and 1:5,000 for iNOS. After being washed with phosphate-buffered saline (PBS), the cells were incubated with a secondary antibody 
containing 1.0\% goat serum for 30 minutes. For labelling, a 1:500 dilution of biotinylated goat antibody against mouse or guinea pig IgG (Vector Laboratories, Burlingame, CA) was used. Bound antibodies were detected by the avidin-biotin-peroxidase complex (ABC) method, using a commercial ABC kit (Vector Laboratories). Observation of the peroxidase activity was made possible by incubation with $0.1 \%$ 3,3'-diaminobenzidine tetrahydrochloride (DAB) in a $50 \mathrm{mM}$ Tris-HCl buffer (pH 7.4) supplemented with $0.02 \% \mathrm{H}_{2} \mathrm{O}_{2}$. The cells were dehydrated in 70 $100 \%$ ethanol, cleared in xylene, and mounted on glass coverslips in Permount (Fisher Scientific, Fair Lawn, NJ) for light microscopic observation.

\section{Western blot analysis}

Cell lysates were diluted 3:1 in sample buffer (187.5 mM Tris-HCl containing $6 \%$ SDS, and 15\% glycerol, 15\% 2-mercaptoethanol) and denatured at $100{ }^{\circ} \mathrm{C}$ for $3 \mathrm{~min}$. Proteins (40 $\mu \mathrm{g}$ for co-cultures, $20 \mu \mathrm{g}$ for astrocyte-enriched cultures per lane) were electrophoresed on a 10\% SDS-polyacrylamide gel and transferred onto a polyvinylidene difluoride membrane (Bio-Rad, Hercules, CA). Nonspecific binding sites were blocked with 5\% non-fat milk for $60 \mathrm{~min}$, and then the membrane was incubated overnight at $4{ }^{\circ} \mathrm{C}$ with one of the following antibodies: polyclonal antibody to 
GLT-1 (1:10000), GLAST (1:8000) (Chemicon, Temecula, CA), and monoclonal antibody to actin (1:2000) (Sigma), and then horseradish peroxidase-labeled anti-guinea pig for GLT-1 and GLAST (1:2000) (Cell Signaling Technology) or anti- mouse IgG antibody for actin (1:2000) (Cell Signaling Technology), respectively. The immunoreactive bands were detected with an enhanced chemiluminescence kit (NEN Life Science Products, Boston, MA). Quantification of the expression levels of GLT-1, GLAST or actin were preformed by densitometric analysis using Scion Image Beta 4.02 for Windows 95, 98, ME, NT 2000 and XP (Scion Corporation).

\section{Oxygen-glucose deprivation}

Cortical cultures were subjected to oxygen-glucose deprivation (OGD) injury using a protocol described previously $(11,12)$. In brief, cultures were placed in an anaerobic chamber and washed two times with a balanced salt solution (BSS: $116 \mathrm{mM}$ $\mathrm{NaCl}, 0.8 \mathrm{mM} \mathrm{MgSO}_{4}, 5.4 \mathrm{mM} \mathrm{KCl}, 1.0 \mathrm{mM} \mathrm{NaH}_{2} \mathrm{PO}_{4}, 26.2 \mathrm{mM} \mathrm{NaHCO}$, $1.8 \mathrm{mM}$ $\mathrm{CaCl}_{2}, 0.01 \mathrm{mM}$ glycine, and $10 \mathrm{mg} / \mathrm{l}$ phenol red) lacking glucose. Near anoxic conditions were achieved using an Anaero-Pack System (Mitsubishi Gas Chemical, Tokyo, Japan). After pre-gassing with $95 \% \mathrm{~N}_{2}-5 \% \mathrm{CO}_{2}$ for at least 5 min to remove residual oxygen, glucose-free BSS was added to the cells, which were then placed in a 
purpose-built sealed chamber containing the deoxygenation reagent (Kenki for Cells, Mitsubishi Gas Chemical, Tokyo, Japan). The catalytic reaction of the reagent resulted in the consumption of $\mathrm{O}_{2}$ and production of $\mathrm{CO}_{2}$. This Anaero-Pack System provided near anaerobic conditions with an $\mathrm{O}_{2}$ concentration of $<1 \%$ and a $\mathrm{CO}_{2}$ concentration of about $5 \%$ within $1 \mathrm{~h}$ of incubation at $37^{\circ} \mathrm{C}$. Cells were exposed to these conditions for a designated period to produce either mild (sublethal) or lethal OGD. To terminate OGD, cultures were carefully washed with glucose (20 mM) containing BSS, and then incubated again in culture medium at $37^{\circ} \mathrm{C}$ in $95 \%$ air-5\% $\mathrm{CO}_{2}$ (reperfusion). Cultures with sham treatment not deprived of oxygen and glucose were placed in BSS containing $20 \mathrm{mM}$ glucose. In some experiments, BSS was prepared with no added calcium salts and the addition of $2 \mathrm{mM}$ EGTA and $1.8 \mathrm{mM}$ $\mathrm{MgCl}_{2}$. For the measurement of the NADH fluorescence, cultures were washed two times with BSS without glucose and phenol red. After washing, glucose-free BSS (containing $50 \mathrm{U} / \mathrm{mL}$ GDH, 2 mM NAD ${ }^{+}, 4 \mathrm{U} / \mathrm{mL}$ GPT, and $2 \mathrm{mM}$ L-Alanine) without phenol red was added to the cultures. The cultures were then placed in a purpose-built sealed chamber made of acrylic resin and glass for transmitting UV light containing the deoxygenation reagent, and examined under a fluorescent microscope (Olympus, IX70, Tokyo, Japan). 


\section{Measurement of the concentration of extracellular glutamate}

The concentration of extracellular glutamate (Glu) was measured using an enzymatic assay $(13,14)$. In the presence of Glu and $\beta$-nicotinamide adenine dinucleotide $\left(\mathrm{NAD}^{+}\right)$, L-glutamic dehydrogenase (GDH) produces $\alpha$-ketoglutarate and $\mathrm{NADH}$, a product that fluoresces when excited at $360 \mathrm{~nm}$. In the presence of $\alpha$-ketoglutarate and L-Alanine, glutamate pyruvate transaminase (GPT) produces L-glutamate. Therefore, GDH (50 U/mL), NAD ${ }^{+}$(2 mM), GPT (4 U/mL), and L-Alanine (2 $\mathrm{mM}$ ) were added to the external solution, and the fluorescence was excited at $360 \mathrm{~nm}$ and detected at $>510 \mathrm{~nm}$ with a fluorescent microscope. Images excited at $360 \mathrm{~nm}$ were acquired with integration times of $5 \mathrm{~s}$ at intervals of $20 \mathrm{~s}$. The extracellular Glu was detected as an increase in NADH fluorescence. This method does not necessarily reflect the quantitative extracellular Glu level, but can detect subtle changes in the concentration of Glu. Fluorescent images were acquired with a cooled CCD camera (C4880-80; Hamamatsu Photonics, Hamamatsu, Japan). An analysis of the acquired images was conducted with an image processing and measuring system (AQUACOSMOS; Hamamatsu Photonics).

\section{Survival rate of neurons}


Neuronal death was analyzed following observation of the nuclear morphology using the fluorescent DNA-binding dyes, Hoechst 33342 (H33342) and propidium iodide (PI). Cells were incubated with these dyes for 15 minutes at $37^{\circ} \mathrm{C}$. Individual nuclei were observed using fluorescent microscopy (Olympus, IX70, Tokyo, Japan) and subsequently analyzed. PI was used to identify nonviable cells. More specifically, an average of 450-500 neurons from random fields were analyzed in each experiment. The survival rate of neurons - meaning the percentage of viable neurons remaining was determined by placing images of nuclear staining on phase-contrast images, and calculating (viable neurons/total neurons before drug treatment) $\times 100$, since some neurons came off the dishes at the time of inspection. At least 4 independent experiments $(n \geq 4)$ were conducted and analyzed.

\section{Chemicals}

$\mathrm{N}^{\mathrm{G}}$-monomethyl-L-arginine (L-NMMA), Bisbenzimide (Hoechst 33342), and propidium iodide (PI) were obtained from Sigma. $\quad \mathrm{N}^{5}$-(1-imino-3-butenyl)-L-ornithine (L-VNIO) was obtained from ALEXIS Japan (Tokyo, Japan). The other chemicals were from Wako Chem. (Tokyo, Japan).

\section{Statistics}


Data were presented as means with standard deviation. Multiple comparisons were conducted using an analysis of variance (ANOVA) with Sheffe's t-test. A $P$-value of less than 0.01 or 0.05 was considered statistically significant. 


\section{RESULTS}

We have recently revealed that the exposure of neuron/astrocyte co-cultures to a sublethal ischemic insult (preconditioning) results in a significant suppression of the rise in the concentration of extracellular glutamate (Glu) during subsequent lethal ischemia, and this suppression is a primary cause of PC-induced neuronal ischemic tolerance (3). In addition, we also demonstrated the possibility that nitric oxide (NO) produced during PC is involved in the development of the PC-induced ischemic tolerance of neurons (10). Therefore, we first investigated whether the PC-induced suppression of the rise in the concentration of extracellular Glu was antagonized by the inhibition of NO production during PC in neuron/astrocyte co-cultures (Fig. 1). Lethal ischemia (90 min OGD) resulted in a marked elevation of the concentration of extracellular Glu (Fig. 1B, $1 \mathrm{E}$ and $1 \mathrm{~F})$. The elevation was significantly decreased by prior PC (60 min OGD) treatment 24h before the onset of lethal OGD (Fig. 1C, 1E and 1F). However, this PC-induced suppression of the rise in extracellular Glu was significantly reversed by the treatment of cultures with $\mathrm{N}^{\mathrm{G}}$-monomethyl-L-arginine (L-NMMA; $1 \mathrm{mM}$ ), a non-specific inhibitor of NO synthase (NOS) during PC (Fig. 1D, 1E and 1F). This 
result suggested that NO produced during PC was crucial to the PC-induced suppression of the rise in the concentration of extracellular Glu during lethal ischemia.

We have recently reported that a PC insult transiently decreases the level of the astrocytic Glu transporter GLT-1, and this decreased expression contributes to the suppression of the rise in extracellular Glu via a reversed uptake of Glu by GLT-1 during lethal ischemia (3). Therefore, we then investigated whether NO is involved in the PC-induced down-regulation of the expression of GLT-1 by conducting immunocytochemical as well as Western blot analyses (Fig. 2). The expression of astrocytic GLT-1 was significantly decreased $24 \mathrm{~h}$ after the PC (Fig. 2A1, 2B1 and 2D2), but this down-regulation was significantly reversed by treatment with L-NMMA (1 mM) during PC (Fig. 2A1, 2B1 and 2D3). In contrast, the expression level of GLAST was not significantly changed (Fig 2A2-2C2).

Our previous study has revealed that PC-induced neuronal ischemic tolerance was attenuated by treatment with a blocker of $\mathrm{Ca}^{2+}$-permeable NMDA receptors during PC (11), suggesting that $\mathrm{Ca}^{2+}$-dependent NOS was involved in the PC-induced tolerance. We thus investigated the expression of NOS isoforms in our neuron/astrocyte co-cultures. The neurons in our co-cultures expressed neuronal NOS (nNOS) (Fig. 3H1), but the distinct expression of neither inducible NOS (iNOS) (Fig. 3H2) nor 
endothelial NOS (eNOS) (Fig. 3H3) was detected in the co-cultures. Therefore, we investigated whether the activation of $\mathrm{Ca}^{2+}$-dependent nNOS during PC was crucial to the development of PC-induced neuronal ischemic tolerance. Lethal (90 min) OGD resulted in a significant death of neurons inspected at $24 \mathrm{~h}$ after the end of the insult (Fig. 3A and 3D). In contrast, exposure of preconditioned cultures to the lethal OGD $24 \mathrm{~h}$ later resulted in a significant increase in the survival rate of neurons (Fig. 3B and 3D). However, this PC-induced protective effect on neurons was significantly antagonized by treatment with vinyl-L-NIO (L-VNIO; $100 \mu \mathrm{M})$, a potent specific inhibitor of nNOS (16), during PC (Fig. 3C and 3D). Furthermore, we investigated whether the production of NO via the activation of nNOS during PC was also critical to the PC-induced down-regulation of GLT-1 by conducting immunocytochemical as well as Western blot analyses. The expression of GLT-1 was decreased $24 \mathrm{~h}$ after the exposure to the prior PC, but the decrease was significantly reversed when the activity of nNOS was inhibited by treatment with L-VNIO $(100 \mu \mathrm{M})$ during PC (Fig. 3E1 and 3F1). In contrast, the expression of GLAST did not change significantly (Fig. 3E2-3G2). These results suggested that nNOS-derived NO produced during PC contributed to the PC-induced decrease in the expression of GLT-1 and to the development of the PC-induced ischemic tolerance of neurons in the neuron/astrocyte 
co-cultures. 


\section{Discussion}

The present study demonstrated that nNOS-derived NO produced during sublethal preconditioning (PC) was crucial for the PC-induced down-regulation of GLT-1 and the increased neuronal resistance to a subsequent severe ischemic insult.

Although numerous studies have reported increased tolerance to ischemia following preconditioning of the mammalian brain with OGD, cortical spreading depression (CSD), or middle cerebral artery occlusion (MCAO), the mechanism underlying this neuroprotection is unclear (17-20). Some studies have reported that neurons themselves acquire resistance against ischemia through preconditioning (19, 20). We have recently reported that the expression of the astrocytic glutamate transporter GLT-1 is transiently decreased after prior PC and contributes to the PC-induced neuronal resistance to subsequent severe ischemic insult, suggesting that the PC-induced changes in glial function; that is, the down-regulation of astrocytic GLT-1 expression, seemed critical to the PC-induced ischemic tolerance of neurons (3). NO is generated in various mammalian tissues, and acts as an intercellular messenger associated with various physiological and pathological events (21). Recently, we have demonstrated that the activity of nitric oxide synthase (NOS) varies with the differentiation of a neuronal cell line (NG108-15 cells), suggesting that NO 
functions as an important signaling molecule in differentiated NG108-15 cells (15). We have also found that NO modulates the propagation of astrocytic $\mathrm{Ca}^{2+}$ waves induced by the local photolysis of a caged $\mathrm{Ca}^{2+}$ ionophore $(22,23)$. In addition, Yun et al. (24) have recently demonstrated that the activation of $\mathrm{p} 21^{\mathrm{RAS}}$ induced by NO derived from nNOS is responsible for the PC-induced tolerance of neurons, suggesting that NO is a key mediator in the process leading to resistance against lethal ischemia. We have previously demonstrated that the enhanced production of $\mathrm{NO}$ is crucial to the development of PC-induced ischemic tolerance of neurons in neuron/astrocyte co-cultures (10). However, the underlying mechanisms responsible for the NO-induced ischemic tolerance have not been fully elucidated. The present study suggested that the production of NO during PC was crucial for the PC-induced down-regulation of GLT-1. The decrease in the expression of GLT-1 seemed crucial for the increased neuronal resistance to a subsequent severe ischemic insult.

We have previously demonstrated that treatment of neuron/astrocyte co-cultures with a blocker of NMDA receptors during PC antagonized the PC-induced ischemic tolerance of neurons (11), suggesting that the activation of $\mathrm{Ca}^{2+}$ permeable NMDA receptors during PC is involved in the development of the PC-induced neuronal ischemic tolerance. In addition, the present study has suggested that the 
$\mathrm{Ca}^{2+}$-dependent nNOS-derived NO produced during PC was critically involved in the PC-induced down-regulation of astrocytic GLT-1, and in the PC-induced ischemic tolerance of neurons. However, the question arises as to what mechanisms are responsible for the NO-induced down-regulation of the glutamate transporter GLT-1. Further studies will be needed to clarify these mechanisms. 


\section{References}

1. Nangopal, K., Dawson, T.M. and Dawson, V.L. 2002. Critical role for nitric oxide signaling in cardiac and neuronal ischemic preconditioning and tolerance. J. Pharmacol. Exp. Ther. 297:474-478.

2. Kitagawa, K., Matsumoto, M., Tagaya, M., Hara, R., Ueda, H., Niinobe, M., Handa, N., Fukunaga, R., Kimura, K., Mikoshiba, K. and Kamada, T. 1990. "Ischemic tolerance” phenomenon found in the brain. Brain Res. 528:21-24.

3. Kosugi, T., Kawahara, K., Yamada, T., Nakajima, T. and Tanaka, M. 2005. Functional significance of the preconditioning-induced down-regulation of glutamate transporter GLT-1 in neuron/astrocyte co-cultures. Neurochem. Res. (in press).

4. Kawahara, K., Hosoya, R., Sato, H., Tanaka, M., Nakajima, T. and Iwabuchi, S. 2002. Selective blockade of astrocytic glutamate transporter GLT-1 with dihydrokainate prevents neuronal death during ouabain treatment of astrocyte/neuron co-cultures. GLIA 40:337-349.

5. Rao, V.L.R., Dogan, A., Todd, K.G., Bowen, K.K., Kim, B-T., Rothstein, J.D. and Dempsey, R.J. 2001. Antisense knockdown of the glial glutamate transporter GLT-1, but 
not the neuronal glutamate transporter EAAC1, exacerbates transient focal cerebral ischemia-induced neuronal damage in rat brain. J. Neurosci. 21:1876-1883.

6. Rothstein, J.D., Dykes-Hoberg, M., Pardo, C.A., Bristol, L.A., Jin, L., Kuncl, R.W., Kanai, Y., Hediger, M.A., Wang, Y., Schielke, J.P. and Welty, D.F. 1996. Knockout of glutamate transporters reveals a major role for astro-glial transport in excitotoxicity and clearance of glutamate. Neuron 16:675-686.

7. Tanaka, K., Watase, K., Manabe, T., Yamada, K., Watanabe, M., Takahashi, K., Iwama, H., Hori, S., Takimoto, M. and Wada, K. 1997. Epilepsy and exacerbation of brain injury in mice lacking the glutamate transporter GLT-1. Science 276:1699-1702.

8. Szatkowski, M. and Attwell, D. 1994. Triggering and execution of neuronal death in brain ischaemia: two phases of glutamate release by different mechanisms. Trends Neurosci. 9:359-365.

9. Szatkowski, M., Barbour, B. and Attwell, D. 1990. Non-vesicular release of glutamate from glial cells by reversed electrogenic glutamate uptake. Nature 348:443-446.

10. Kawahara, K., Yanoma, J., Tanaka, M., Nakajima, T. and Kosugi, T. 2004. Nitric oxide produced during ischemia is toxic but crucial to preconditioning-induced ischemic tolerance of neurons in culture. Neurochem. Res. 29:797-804. 
11. Kawahara, K., Kosugi, T., Tanaka, M., Nakajima, T. and Yamada, T. 2005. Reversed operation of glutamate transporter GLT-1 is crucial to the development of preconditioning-induced ischemic tolerance of neurons in neuron/astrocyte co-cultures. GLIA 49:349-359.

12. Kawahara, K, Abe, R., Yamauchi, Y. and Kohashi, M. 2002. Fluctuations of contraction rhythm during simulated ischemia/reperfusion in cultured cardiac myocytes from neonatal rats. Biol. Rhythm Res. 33:339-350.

13. Innocenti, B., Parpura, V. and Haydon, P.G. 2000. Imaging extracellular waves of glutamate during calcium signaling in cultured astrocytes. J. Neurosci. 20:1800-1808.

14. Maguire, G., Simko, H., Weinreb, R.N. and Ayoub, G. 1998. Transport-mediated release of endogenous glutamate in the vertebrate retina. Pflügers Arch. 436:481-484.

15. Kawahara, K., Saitoh, M., Nakajima, T., Tanaka, M., Sato, H., Tojima, T., and Ito, E. 2002. Increased resistance to nitric oxide cytotoxicity associated with differentiation of neuroblastoma-Glioma hybrid (NG108-15) cells. Free Radical Res. 36:545-554.

16. Babu, B.R. and Griffith, O.W. 1998. $\mathrm{N}^{5}$-(1-Imino-3-butenyl)-L-ornithine: A neuronal isoform selective mechanism-based inactivator of nitric oxide synthase. $J$. Biol.Chem. 273:8882-8889.

17. Douen, G.A., Akiyama, K., Hogan, J.M., Wang, F., Dong, L., Chow, K.A. and 
Hakim, A. 2000. Preconditioning with cortical spreading depression decreases intraischemic cerebral glutamate levels and down-regulates excitatory amino acid transporters EAAT1 and EAAT2 from rat cerebral cortex plasma membranes. $J$. Neurochem. 75:812-818.

18. Kato, H., Liu, Y., Araki, T. and Kogure, K. 1992. MK-801, but anisomycin, inhibits the induction of tolerance to ischemia in the gerbile hippocampus. Neurosci. Lett. 139:118-121.

19. McLaughlin, B., Hartnett, K.A., Erhardt, J.A., Legos, J.J., White, R.F., Barone, F.C. and Aizenman, E. 2003. Caspase 3 activation is essential for neuroprotection in preconditioning. Proc. Natl. Acad. Sci. U S A 21:715-720.

20. Nakajima, T., Iwabuchi, S., Miyazaki, H., Okuma, M., Inanami, O., Kuwabara, M., Nomura, Y. and Kawahara, K. 2002. Relationship between the activation of CREB and ischemic tolerance in the penumbra region of rat cerebral cortex. Neurosci. Lett. 331:13-16.

21. Nathan, C. 1992. Nitric oxide as a secretory product of mammalian cells. FASEB J. 6:3501-3604.

22. Iwabuchi, S., Kawahara, K., Makisaka, K., and Sato, H. 2002. Photolytic flash-induced intercellular calcium waves using caged calcium ionophore in cultured 
astrocytes from newborn rats. Exp. Brain. Res. 146:103-116.

23. Kawahara, K. and Iwabuchi, S. 2003. Possible involvement of nitric oxide in the modulation of photolytic flash-induced intercellular calcium waves in cultured astrocytes. Neurosci. Res. Commun. 32:189-196.

24. Yun, H.Y., Gonzalez-Zulueta, M., Dawson, V.L., and Dawson, T.M. 1998. Nitric oxide mediates N-methyl-D-aspartate receptor-induced activation of p21 ${ }^{\text {ras }}$. Proc. Natl. Acad. Sci. USA 95:5773-5778. 


\section{Figure captions}

\section{Fig. 1}

Nitric oxide (NO) produced during preconditioning (PC) involved in the PC-induced suppression of the elevation in the extracellular concentration of glutamate (Glu) during oxygen/glucose deprivation (OGD). Figures A1 and A2 show the results of immunostaining of the cultures using anti-MAP-2 (A1) and anti-GFAP (A2) antibodies, indicating that the cultures were mixed. Photomicrographs B1, C1, and D1 show phase-contrast images of the mixed neuron/astrocyte cultures. Figures B2-B3, C2-C3, and D2-D3 illustrate a pseudo-color representation of NADH fluorescence reflecting the extracellular concentration of Glu when the sham-treated cultures (B2, B3), the cultures preconditioned $24 \mathrm{~h}$ before (C2, C3), and the cultures preconditioned with L-NMMA (1 mM) (D2, D3), were exposed to OGD, respectively. The fluorescence intensity increases from dark blue to red through yellow. Figure E shows a time course of the change in the NADH fluorescence ratio during OGD in the sham-treated cultures (blue), in the preconditioned cultures (orange), and in the cultures preconditioned with L-NMMA treatment (red), respectively. The OGD begins at 0 
min. Vertical bars indicate + SD. Neither neurons nor astrocytes were degenerated at all at 90 min after the start of OGD (lethal OGD). Figure F shows a statistical comparison of the relative fluorescence intensity 90 min after the onset of OGD. Data are expressed as the mean $+\mathrm{SD}\left(\mathrm{n}=4\right.$ different cultures). $\quad{ }^{* *} \mathrm{p}<0.01{ }^{*} \mathrm{p}<0.05$. The scale bars indicate $100 \mu \mathrm{m}$ in A and $200 \mu \mathrm{m}$ in B-D, respectively. Abbreviations: F0, fluorescence intensity just before the onset of OGD; F, fluorescence intensity during OGD; PC, preconditioned with 60 min OGD 24 h before the experiment; L-NMMA, treated with L-NMMA during PC.

Fig. 2

Treatment with an inhibitor of NOS during preconditioning (PC) antagonized the PC-induced down-regulation of the expression of glutamate transporter GLT-1. Western blot analyses of GLT-1, GLAST and actin in the preconditioned cultures (24 h after the PC insult), the cultures preconditioned with L-NMMA (1 mM) and the sham-treated cultures. In figures A1 and A2, $40 \mu \mathrm{g}$ of protein from different cultures was loaded in each lane. Lanes 1 and 2 show GLT-1, GLAST and actin detected in the sham-treated cultures. Lanes 3 and 4 show 24h after the PC. Lanes 5 and 6 show 24 
h after the PC with L-NMMA, respectively. Figures B1 and B2 show the quantitative analysis of GLT-1 and GLAST expression, respectively. Figures C1 and C2 show the quantitative analysis of actin expression from the same sample. The expression levels are normalized with the mean expression level in the sham-treated cultures. Data are expressed as the mean $+\mathrm{SD}\left(\mathrm{n}=4\right.$ different cultures). ${ }^{* *} \mathrm{p}<0.01 .{ }^{*} \mathrm{p}<0.05$. An immunocytochemical analysis of the co-cultures using anti-GLT-1 antibody revealed that the PC insult down-regulated astrocytic GLT-1 24 h after the end of the PC (D2), but the PC-induced down-regulation was antagonized by the treatment with L-NMMA (1 mM) (D3). Figure D1 shows GLT-1 expression in the sham-treated cultures. GLT-1-positive astrocytes were identified just underneath the aggregate of neurons similar to our previous observations (4). GLT-1-positive neurons were not detected. The scale bar indicates $200 \mu \mathrm{m}$.

Fig. 3

Activation of nNOS during PC is crucially involved in the development of PC-induced neuronal ischemic tolerance and in the down-regulation of astrocytic glutamate transporter GLT-1. Photomicrographs A, B and C are images $24 \mathrm{~h}$ after the 
end of OGD when the sham-treated cultures (A), the preconditioned cultures (B), and the preconditioned cultures treated with L-VNIO $(100 \mu \mathrm{M})(\mathrm{C})$ were exposed to lethal ischemia (LI) for 90 min OGD, respectively. Photomicrographs A1, B1 and C1 show the cultures before the exposure to LI, whereas A2, B2 and C2 illustrate their state $24 \mathrm{~h}$ after the exposure, respectively. Cell nuclei were stained with bisbenzimide (Hoechst 33342) and propidium iodide (PI) (A3, B3 and C3). Red nuclei in A3, B3 and C3 indicate PI-positive dead neurons. Figure D shows a statistical comparison of the survival rate of neurons. PC-induced neuronal tolerance was significantly attenuated by treatment with L-VNIO during PC. Western blot analyses of GLT-1, GLAST and actin in the preconditioned cultures (24 h after the PC insult), cultures preconditioned with L-VNIO, and the sham-treated cultures. In Figures E1 and E2, $40 \mu \mathrm{g}$ of protein from different cultures was loaded in each lane. Lanes 1 and 2 show GLT-1, GLAST and actin detected in the sham-treated cultures. Lanes 3 and 4 show them $24 \mathrm{~h}$ after the preconditioning. Lanes 5 and 6 show them $24 \mathrm{~h}$ after the preconditioning treated with L-VNIO during PC. Figures F1 and F2 show the quantification of GLT-1 and GLAST expression, respectively. Figures G1 and G2 show the quantification of actin expression from the same sample. The expression levels are normalized with the mean expression level in the sham-treated cultures. Data are expressed as the mean + SD 
( $\mathrm{n}=4$ different cultures). $\quad * * \mathrm{p}<0.01 . \quad * \mathrm{p}<0.05$. Figures H1-H3 show the results of immunocytochemical analyses of the co-cultures using anti-nNOS (H1), anti-iNOS (H2), and anti-eNOS (H3) antibodies. The analysis revealed that neurons in the co-cultures expressed nNOS, but not iNOS and eNOS. The expression pattern of NOS isoforms was not changed by the sublethal PC (10), suggesting that nNOS-derived NO was crucial to the PC-induced down-regulation of GLT-1. The scale bar indicates 200 $\mu \mathrm{m}$. 

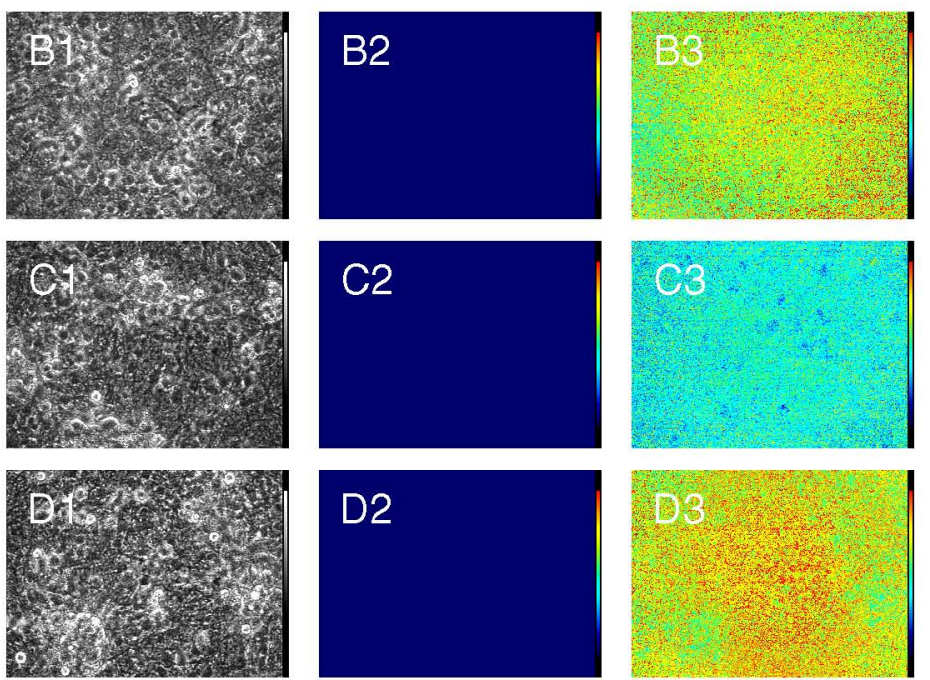

E

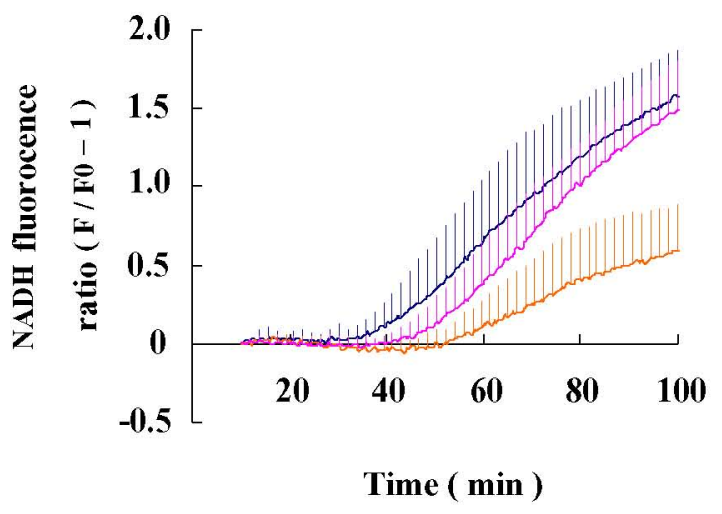

Time ( min )

F

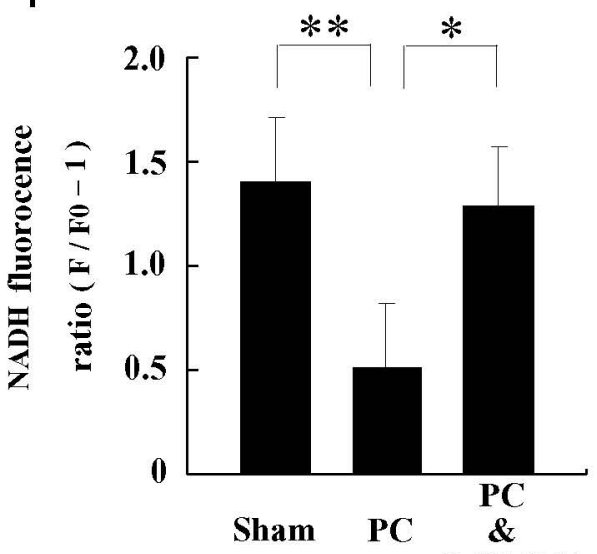


A1

\section{$\begin{array}{llllll}1 & 2 & 3 & 4 & 5 & 6\end{array}$}

GLT-1

actin

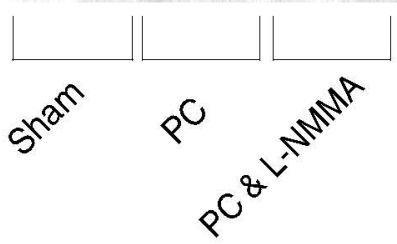

A2

\section{GLAST}

actin
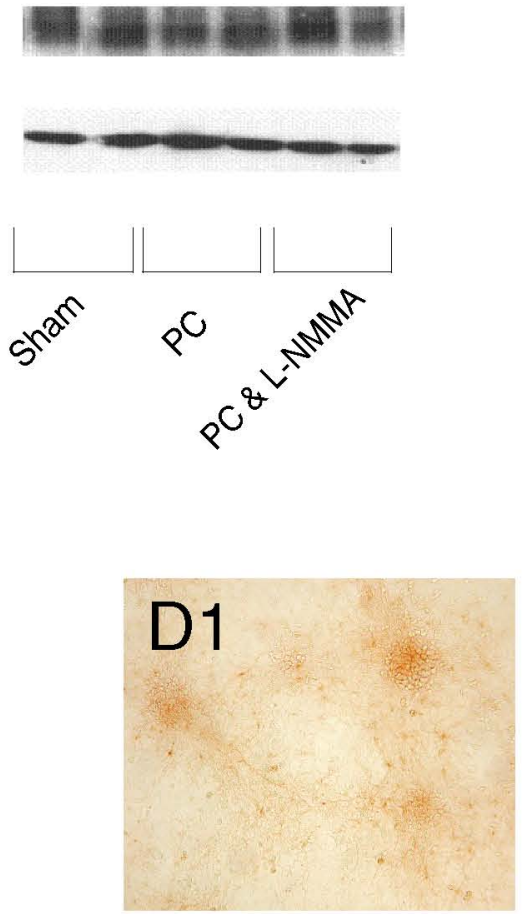

B1
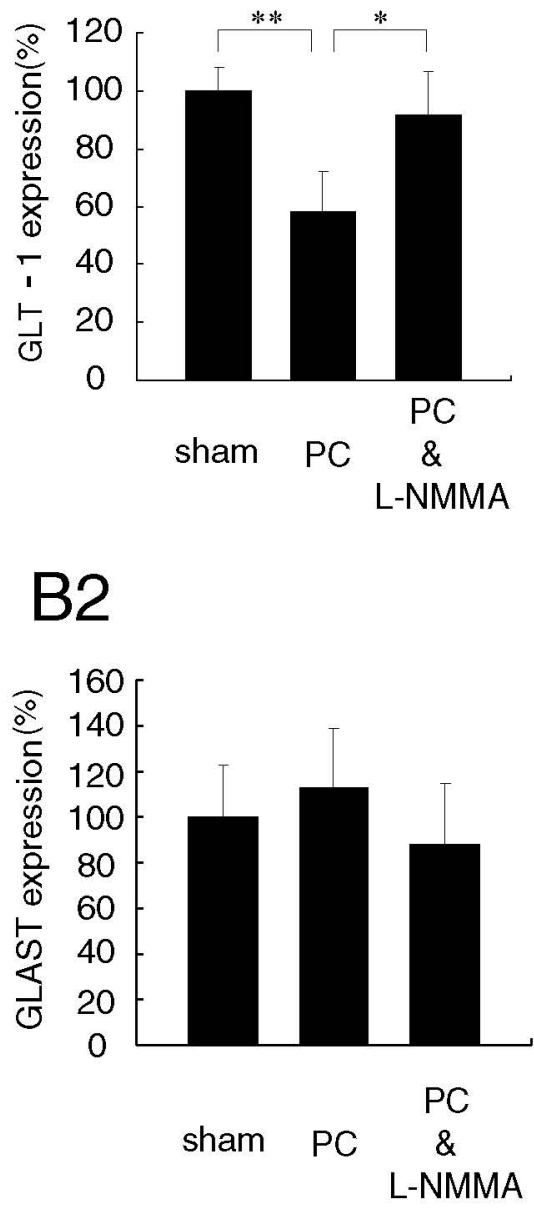

C1

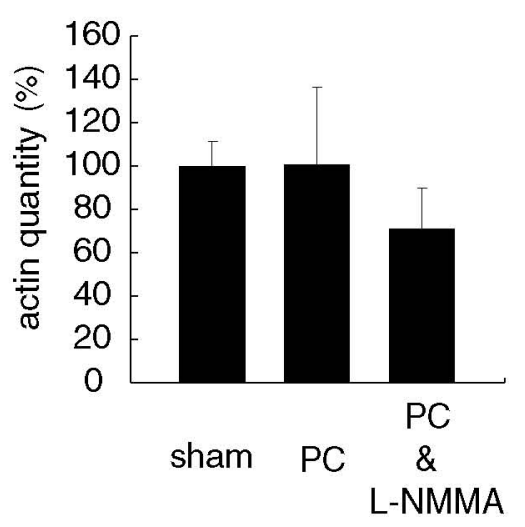

C2

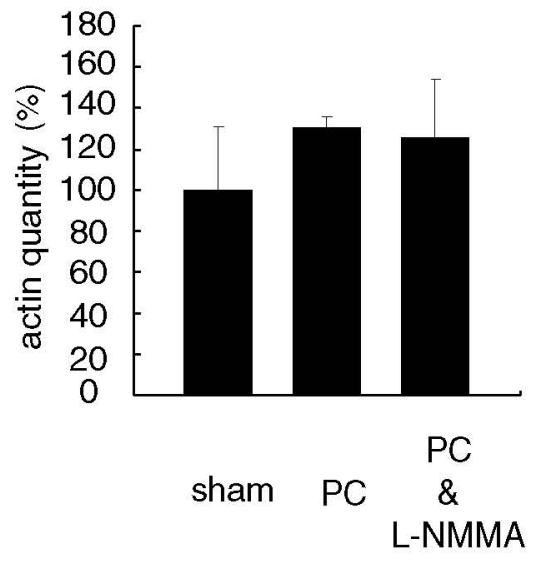

D3 

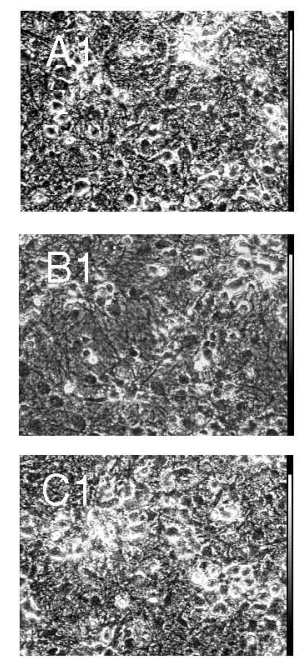

E1

GLT-1

\section{$\begin{array}{llllll}1 & 2 & 3 & 4 & 5 & 6\end{array}$}

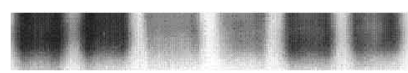

actin

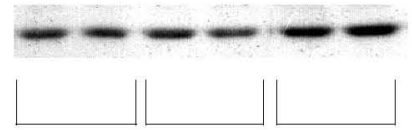

$s^{n^{n}} \quad p^{0}$

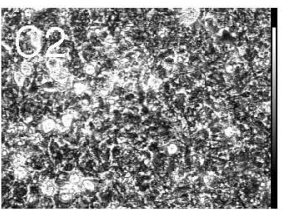

F1

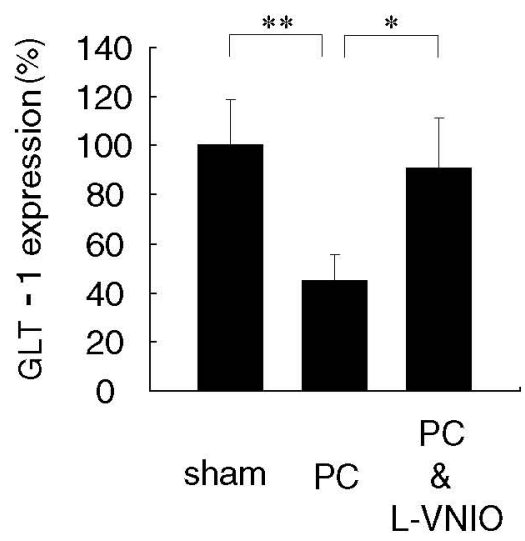

F2

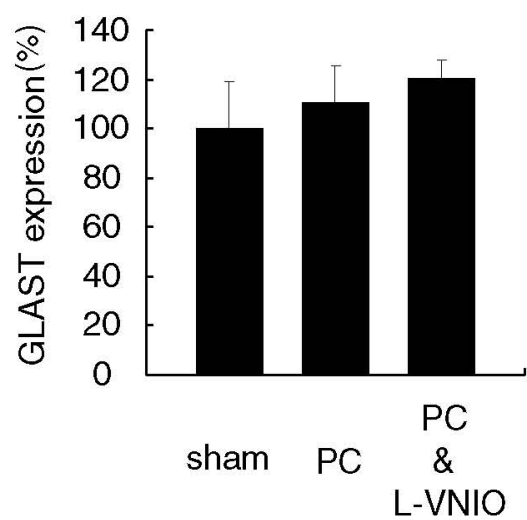

E2

actin<smiles>[13CH3][13CH3]</smiles>

GLAST

actin
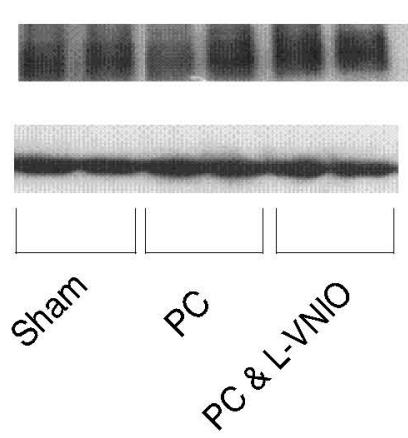

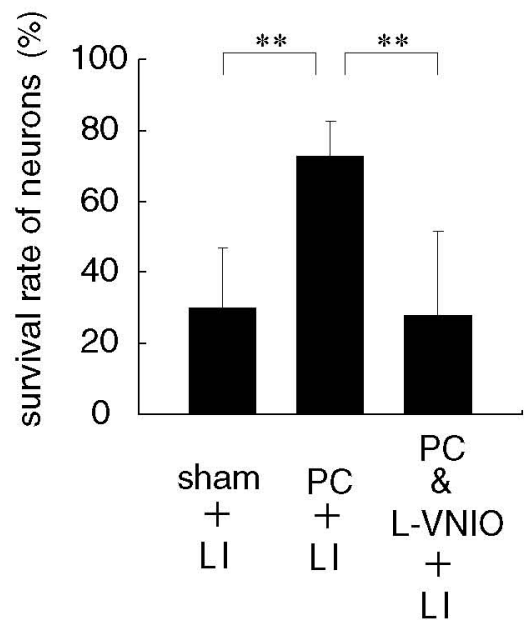

G1

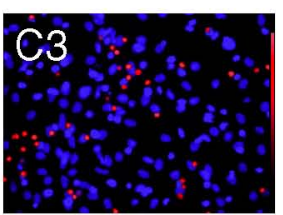

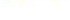
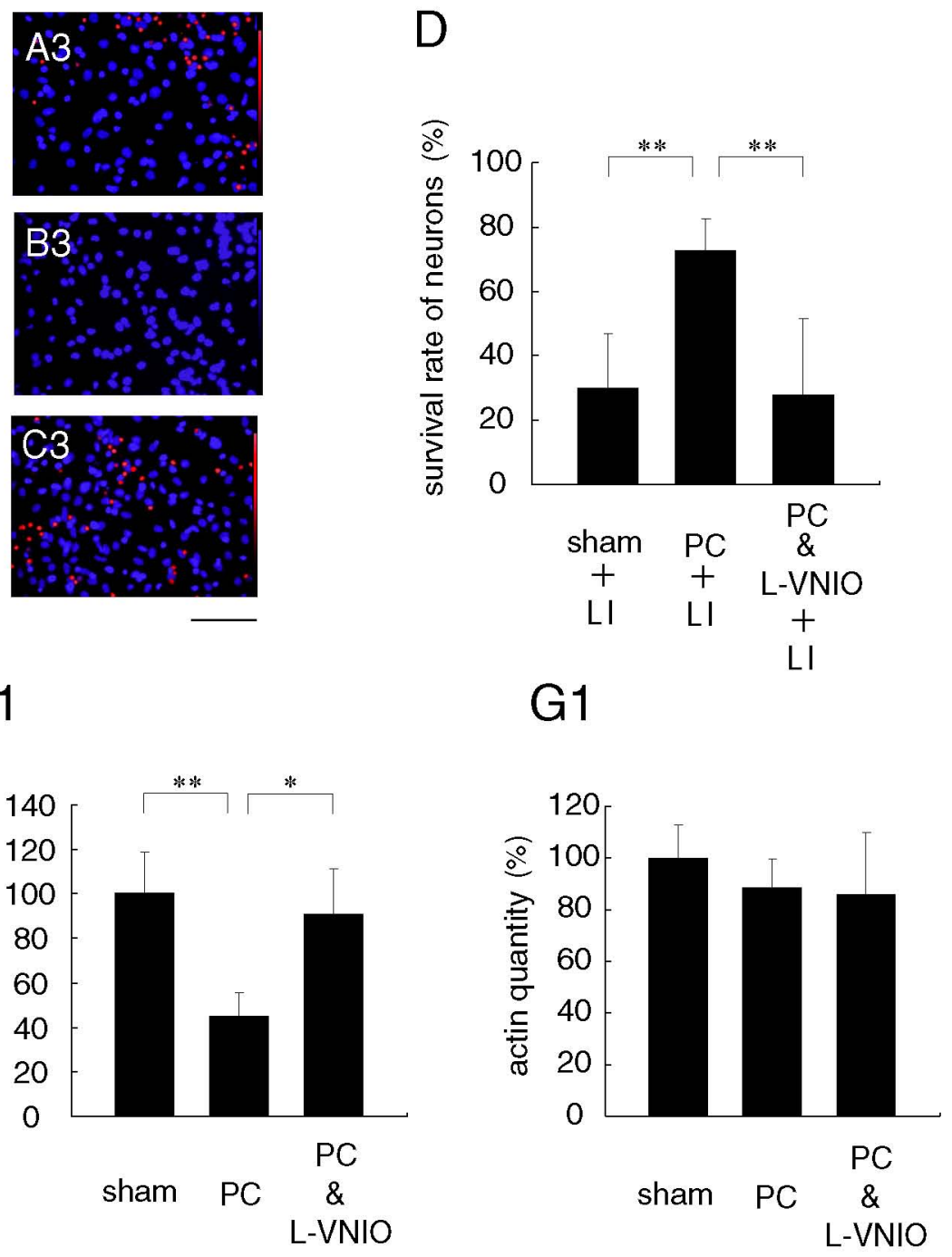

G2

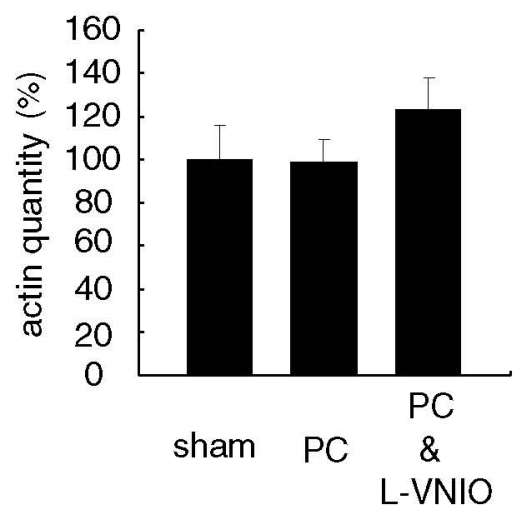

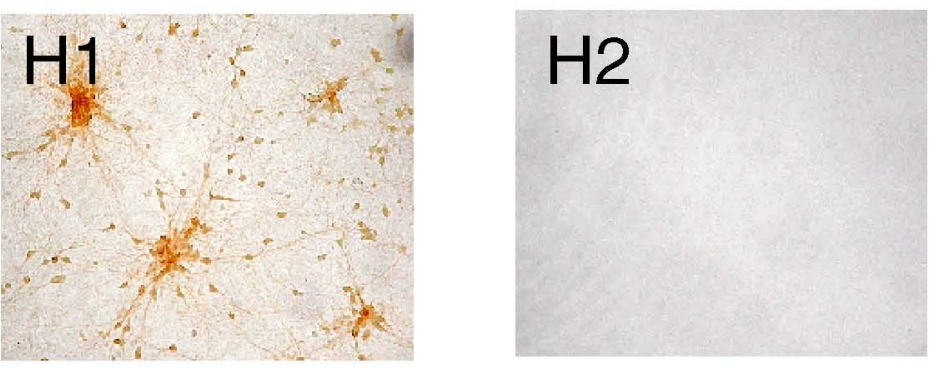

